\title{
Erratum to: Tumor burden evaluation in NF1 patients with plexiform neurofibromas in daily clinical practice
}

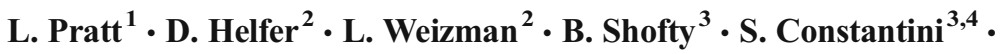 \\ L. Joskowicz ${ }^{2}$ D. Ben Bashat ${ }^{4,5}$ • L. Ben-Sira ${ }^{1,4}$
}

Published online: 12 April 2015

(C) Springer-Verlag Wien 2015

Erratum to: Acta Neurochir

DOI 10.1007/s00701-015-2366-z

The original version of this paper unfortunately contained error. Figure $1 \mathrm{c}$ is incorrect and should be replaced by the following Fig. 1c:

The online version of the original article can be found at http://dx.doi.org/ 10.1007/s00701-015-2366-z.

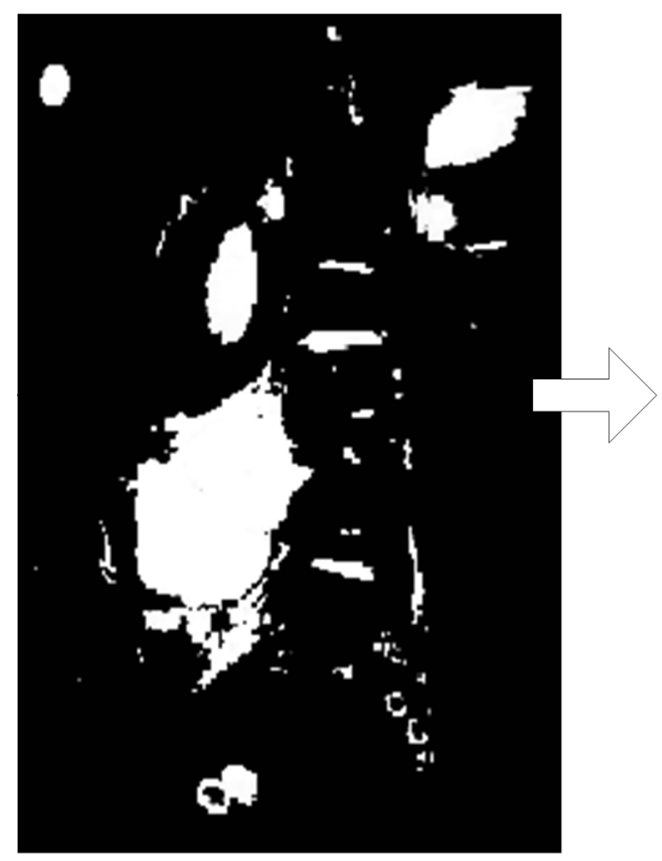

(c)

L. Pratt

litalpratt@gmail.com

Imaging Division, Tel Aviv Sourasky Medical Center, 6 Weizmann Street, Tel Aviv 64239, Israel

2 School of Engineering and Computer Science, The Hebrew University of Jerusalem, Jerusalem, Israel

3 Department of Pediatric Neurosurgery, and the Gilbert International Neurofibromatosis Center, Tel Aviv Sourasky Medical Center, Tel Aviv, Israel

4 Sackler Faculty of Medicine, Tel Aviv University, Tel Aviv, Israel

5 Functional Brain Center, Tel Aviv Sourasky Medical Center, Tel Aviv, Israel 